\title{
Geometric Interpretation of the Uncertainty Principle
}

\author{
Pavel A. Stabnikov
}

Institute of Inorganic Chemistry, Novosibirsk, Russia

Correspondence to: Pavel A. Stabnikov, stabnik@niic.nsc.ru

Keywords: Uncertainty Principle, Unattainable Limits, Geometry of Infinitely Small, Wave-Particle Duality

Received: March 1, $2019 \quad$ Accepted: May 27, $2019 \quad$ Published: May 30, 2019

Copyright (C) 2019 by authors and Scientific Research Publishing Inc.

This work is licensed under the Creative Commons Attribution International License (CC BY 4.0).

http://creativecommons.org/licenses/by/4.0/

\section{Open Access}

\section{ABSTRACT}

The problems of unattainable infinity and infinitesimal are discussed. Limitations connected with the absolute zero of temperature and the maximal velocity are considered, as well as the consequences of these limitations. A geometric approach is proposed as an alternative to the wave-particle duality to explain the anomalous motion of micro objects. The basis of the geometric approach is a comparison between two geometries differing from each other in the metric of infinitesimal. The interconnection of these geometries is possible through the direct and inverse Weierstrass transformation. The application of this transformation allows one to explain diffraction effects.

According to Heisenberg's uncertainty principle, the geometry of the micro world does not differ from our usual macro geometry, but the uncertainty of simultaneous determination of such corpuscular characteristics as the coordinate $(x)$ and impulse $(p)$, time and energy etc. Any pair of these characteristics is bound through the relation $\Delta x \Delta p \geq h$, where $h$ is M. Planck's constant. This relation shows that the smaller is the uncertainty of one value $(x$ or $p)$, the larger is the uncertainty of another one. It is possible that one of the variables has an exact value (for example, $\Delta x=0$ ), while the other variable turns out to be perfectly uncertain ( $\Delta p$ is equal to infinity). This approach is generally accepted [1]. However, a different interpretation is possible, when the space of micro particles differs from the customary geometry by the increased value of infinitesimal (a point). In the geometry of micro objects, the description of the motion of objects is usual, but indistinctness arises as a result of the transformation of the motion information into the geometry with smaller infinitesimal value (to the macro level). With this approach, the difference between the objects in two geometries is reduced to different sharpness of figures, or images. This is how image blur arises during the transformation of figures into the macro geometry on the basis of the data of micro geometry. Heisenberg's indeterminacy principle follows from this comparison in the natural manner. The main thing is that this geometric approach is more fundamental (it is based on understandable geometric statements) than the wave-particle duality relying on two incommensurable notions (particle and wave). However, this approach causes a cardinal change of the conventional idea of infinitesimal. In other words, it is proposed to endow the finite value (fuzzy point) geometrically with the attribute of infi- 
nitely small with the effect of unattainability. However, it should be noted that the examples of the attribute of unattainability of finite values are known in physics: the absolute zero of temperature (Kelvin) and the velocity of light.

In earlier representations, body temperature could take on any values from $-\infty$ to $+\infty$. However, Lord Kelvin proposed to transfer the minimal temperature value to the finite value $\left(-275.15^{\circ} \mathrm{C}\right)$ and accept this value as zero. Then many thermodynamic equations can be written down in a substantially simpler manner. In fact, this approach is the transfer of an infinitely far away value ( $-\infty$ for temperature) to the zero of Kelvin scale. This approach automatically transfers also the unattainability of an infinitely remote point to the zero of Kelvin scale, at which this temperature value becomes unattainable. An additional effect of this transfer is a decrease in the thermal capacity of bodies almost to zero when approaching the zero of Kelvin scale. Otherwise unattainability of the zero of Kelvin scale would be impossible. According to this approach, a body may be cooled so that its temperature will approach the zero of Kelvin scale but it is impossible to reach this finite temperature value. This is the manifestation of the attribute of unattainability.

Another value which is finite but unattainable for anybody with nonzero mass is the velocity of light. Ancient philosophers (with rare exception) thought that the velocity of light is infinite. The finiteness of the velocity of light was established for the first time in 1676 by O. Roemer on the basis of the differences in the times of satellite shadowing by Jupiter between the cases when the Earth moves closer or away from Jupiter. Later this value was determined more precisely many times with the help of special devices allowing more accurate determination of distance and time. The General Assembly of the International Committee on Numerical Data for Science and Technology (CODATA recommended values of the fundamental physical constants: 2010), evaluated all the available data for the velocity of light in vacuum and accepted the constant value equal to $299,792,458 \mathrm{~m} / \mathrm{s}$ [2].

In 1905 A. Einstein developed his Special Theory of Relativity (SR) to achieve agreement between the laws of classical mechanics and electrodynamics. According to this theory, the velocity of light measured in any inertial reference system is the same and does not depend on the motion of the system and the radiator. In classical mechanics, only the infinite velocity possessed the attribute of unattainability because it is impossible to achieve infinite velocity. According to the SR, the maximal velocity is the velocity of light, with the conservation of unattainability attribute. The consequences of unattainability attribute for the velocity of light are the following statements: relativistic law of velocity composition, time dilation and reduction of the linear dimensions of a moving body with respect to a chosen inertial system. Otherwise unattainability of the finite velocity of light would be impossible. According to the SR, the velocity of any body may be arbitrary close to the velocity of light but this limit cannot be exceeded.

These two examples were described to illustrate the efficiency and simplicity of the description of nature with the help of so unusual transformations of infinitely far. The major attribute of infinitely large is unattainability because nothing can be larger than infinitely large. From the point of view of philosophy, not only infinitely large but also infinitely small possessed the attribute of unattainability. For instance, mathematical zero is an inverse value of infinitely large value. The attribute of unattainability of infinitely little is traced in the aporias of Zeno, an ancient Greece philosopher (for example, about Achilles and the tortoise). Unattainability of infinitely small is used in higher mathematics to prove many theorems. Antagonism and interconnection of infinitely large and infinitely small allow assuming possible usefulness of the idea to give the attribute of unattainability to a small value, similar to the unattainability of infinitely small. It was demonstrated in $[3,4]$ that this approach allows theoretical explanation of quantum effects during the transformation of images from one geometry into another.

The best approach to provide interconnection of the images of two geometries with different values of infinitely small is the direct and inverse integral Weierstrass transformation:

$$
F(t)=\frac{1}{\sqrt{4 \pi}} \int_{-\infty}^{+\infty} \mathrm{e}^{\frac{-(x-t)^{2}}{4}} f(x) \mathrm{d} x, \quad f(x)=\frac{1}{i \sqrt{4 \pi}} \int_{s-i \infty}^{s+i \infty} \mathrm{e}^{\frac{-(t-x)^{2}}{4}} F(t) \mathrm{d} t
$$


At present, computers allow numerical modeling with the help of the direct and inverse Weierstrass transformations. Some results of modeling involving a bell-shaped function $\mathrm{e}^{-A^{2} x^{2}}$, which is close to the Gauss normal distribution functions, are described in $[3,4]$.

\section{CONFLICTS OF INTEREST}

The author declares no conflicts of interest regarding the publication of this paper.

\section{REFERENCES}

1. Feynman, R., Leighton, R. and Sands, M. (1963) Feynman Lectures of Physics. Vol. 3, Moscow, 250.

2. Mohr, P.J., Taylor, B.N. and Newell, D.B. (2012) CODATA Recommended Values of the Fundamental Physical Constants: 2010. Reviews of Modern Physics, 84, Article ID: 1527.

https://doi.org/10.1103/RevModPhys.84.1527

3. Stabnikov, P.A. (2018) Frames in Which Matter Develops. Palmarium Academic Publishing. (In Russian)

4. Stabnikov, P.A. and Babailov, S.P. (2019) Types of Interactions and Material Islands of Stability: From the Micro World to the Universal Scale. IIC SB RAN, Novosibirsk. (In Russian) 